\title{
Produtividade de laranja correlacionada com atributos químicos do solo visando a zonas específicas de manejo ${ }^{1}$
}

\author{
Nídia Raquel Costa ${ }^{2}$, Morel de Passos e Carvalho ${ }^{3}$, \\ Edjair Augusto Dal Bem³ ${ }^{3}$ Flávio Carlos Dalchiavon ${ }^{4}$, Rudgen Rodrigues Caldas ${ }^{3}$
}

\begin{abstract}
Orange yield correlated with soil chemical attributes aiming specific management zones

Precision agriculture determines the exact crop management, based on the mapping of specific soil management zones. Thus, this study aimed to characterize the concept of specific soil management zone by using spatial correlations and ordinary cokriging between Valência orange yield and soil attributes, aiming to select the soil attribute that is more efficiently related to the increase in yield, in a geostatistical grid with 120 sampling points, in 1.65 ha. The soil attributes surveyed were: $\mathrm{P}, \mathrm{OM}, \mathrm{pH}, \mathrm{K}^{+}, \mathrm{Ca}^{2+}, \mathrm{Mg}^{2+}, \mathrm{H}^{+}+\mathrm{Al}^{3+}, \mathrm{S}, \mathrm{T}$ and $\mathrm{V} \%$. The data were submitted to descriptive analysis, Pearson's correlations end geostatistical analysis. Thus, the orange yield resulted in significant ordinary cokrigings, for both the $\mathrm{pH}$ and soil $\mathrm{Mg}$ content evaluated. This technique allowed the creation of two specific zones of soil $\mathrm{pH}$ management closely associated to that yield. The obtaining of two specific management zones ratified the importance of precision agriculture, concerning the increased orange yield, because by keeping the two specific management zones obtained, the global yield of the area would be $16.91 \mathrm{t} \mathrm{ha}^{-1}$. However, by providing optimal conditions of soil $\mathrm{pH}$ to the one with the highest acidity level, it would be possible to obtain an increase of $7.58 \mathrm{tha}^{-1}$ in fruit yield, reaching the global average of $24.49 \mathrm{tha}^{-1}$.
\end{abstract}

KEY-WORDS: Citrus sinensis (L.) Osbeck; precision agriculture; geostatistics; soil management and conservation.

\section{INTRODUÇÃO}

A agricultura de precisão determina o exato manejo da lavoura, tendo como base o mapeamento de zonas específicas de manejo do solo. No Brasil, a agricultura de precisão encontra-se em fase de adoção, na qual o agricultor tenta elaborar

\section{RESUMO}

A agricultura de precisão determina o exato manejo da lavoura, tendo como base o mapeamento de zonas específicas de manejo do solo. Assim, tentou-se caracterizar o conceito de zona específica de manejo do solo, pelo emprego de correlações espaciais, usando a cokrigagem ordinária entre a produtividade de laranja Valência e atributos do solo, objetivando selecionar o atributo do solo que mais eficientemente fosse relacionado com o aumento da referida produtividade, em malha geoestatística com 120 pontos amostrais, em 1,65 ha. Os atributos do solo pesquisados foram: $\mathrm{P}, \mathrm{MO}, \mathrm{pH}, \mathrm{K}^{+}, \mathrm{Ca}^{2+}, \mathrm{Mg}^{2+}, \mathrm{H}^{+}+\mathrm{Al}^{3+}, \mathrm{S}, \mathrm{T}$ e $\mathrm{V} \%$. Foram aplicadas aos dados a análise descritiva, correlações de Pearson e análise geoestatística. Assim, a produtividade de laranja resultou em significativas cokrigagens ordinárias, tanto com o $\mathrm{pH}$ como com o teor de $\mathrm{Mg}$ do solo pesquisado. Essa técnica possibilitou a criação de duas zonas específicas de manejo do $\mathrm{pH}$ do solo intimamente associadas à referida produtividade. A obtenção de duas zonas específicas de manejo ratificou a importância da agricultura de precisão, frente ao aumento da produtividade de laranja, pois, mantendo-se as duas zonas específicas de manejo obtidas, a produtividade global da área seria de 16,91 tha-1. Contudo, proporcionando-se condições ótimas de $\mathrm{pH}$ do solo àquela de maior acidez, poder-se-ia obter incremento de 7,58 tha-1 na produtividade de frutos, alcançando a média global de $24,49 \mathrm{t} \mathrm{ha}^{-1}$.

PALAVRAS-CHAVE: Citrus sinensis (L.) Osbeck; agricultura de precisão; geoestatística; manejo e conservação do solo.

mapas de produtividade agrícola que possuam elevada correlação espacial com os mapas de nutrientes vegetais. Seus maiores benefícios são a redução do custo de produção, devido ao menor gasto com insumos, e o aumento da produtividade agrícola (Tschiedel \& Ferreira 2002, Molin et al. 2007).

1. Trabalho recebido em out./2013 e aceito para publicação em out./2014 ( $\mathrm{n}^{\mathrm{o}}$ registro: PAT 27101).

2. Universidade Camilo Castelo Branco (Unicastelo), Fernandópolis, SP, Brasil.E-mail: nidiarcosta@gmail.com.

3. Universidade Estadual Paulista "Júlio de Mesquita Filho" (Unesp), Faculdade de Engenharia, Departamento de Fitossanidade, Engenharia Rural e Solos, Ilha Solteira, SP, Brasil.E-mails: morel@agr.feis.unesp.br, edjairflorestal@hotmail.com, rcaldas_rudgen@gmail.com.

4. Instituto Federal de Educação, Ciência e Tecnologia de Mato Grosso (IFMT), Campo Novo do Perecis, MT, Brasil. E-mail: flavio.dalchiavon@cnp.ifmt.edu.br. 
Assim, tendo-se como exemplo a utilização de um determinado adubo, seria possível aplicá-lo com taxas variáveis, ou seja, somente nos locais em que fosse necessário. Nas áreas onde ocorressem os menores teores nutricionais, normalmente associadas às mais baixas produtividades agrícolas, deveriam ser empregadas as mais elevadas doses de adubo, sendo o inverso verdadeiro. Portanto, esse é o aspecto que decisivamente diferencia a agricultura de precisão da convencional, ao efetuar a aplicação do adubo com base na média global da área cultivada.

Ageoestatística analisa a dependência espacial de dados georreferenciados, aos quais é ajustado o semivariograma, representado pelo gráfico da semivariância, em função das distâncias entre observações. A partir dele, pode-se confeccionar um mapa, por meio da técnica de krigagem, para cada atributo pesquisado, do solo ou da planta, que representa a variabilidade espacial dos dados. Contudo, havendo afinidade entre as dependências espaciais de dois atributos quaisquer, modelada pelo semivariograma cruzado, pode-se obter um mapa por meio da técnica de cokrigagem, para o atributo principal, de difícil obtenção e de maior interesse, em função do atributo secundário, normalmente de muito fácil obtenção (Molin et al. 2007, Lima et al. 2010, Montanari et al. 2010, Dalchiavon et al. 2011a e 2011b). Poder-se-ia, então, a partir do atributo secundário, obter as zonas específicas de manejo do solo, para o atributo primário.

A citricultura é uma das mais destacadas atividades da agroindústria brasileira, caracterizada como um setor altamente organizado e competitivo, responsável por $60 \%$ da produção mundial de suco de laranja (Brasil 2012). A pesquisa citrícola, que, normalmente, envolve os países produtores de laranja, tem envidado esforços para propor metodologias voltadas à criação de zonas específicas de manejo, visando ao aumento de produtividade dos pomares. Assim, há um número bem expressivo de pesquisadores propondo a criação de mapas de produtividade do fruto (Farias et al. 2003, Molin \& Mascarin 2007), como, também, tentando obter zonas específicas de manejo pela cokrigagem da produtividade de frutos, em função dos teores nutricionais do solo (Molin et al. 2007).

Nesse contexto, na maioria dos trabalhos consultados, evidenciou-se que a geoestatística têm se tornado uma ferramenta extremamente relevante para auxiliar os programas de agricultura de precisão, pela geração de mapas de produtividade. Neles, é possível delimitar áreas de manejo diferenciado, garantindo culturas com elevada produtividade e, principalmente, propor o uso racional dos recursos naturais e dos insumos, aspectos decisivos que podem proporcionar maior sustentabilidade do setor.

Este estudo objetivou caracterizar zonas específicas de manejo do solo, empregando correlações espaciais e de Pearson, entre a produtividade de frutos de laranja e alguns atributos químicos do solo, para fins de fertilidade, no sentido de se indicar aquele que esteja mais eficientemente relacionado com o aumento da produtividade.

\section{MATERIAL E MÉTODOS}

O estudo foi realizado entre agosto e dezembro de 2011, no Sítio Santa Rita (20¹9'52”S, 5055'30”W e altitude de $365 \mathrm{~m}$ ), localizado no município de Nova Canaã Paulista (SP). O tipo climático é $\mathrm{A}_{w}$, caracterizado por possuir estação chuvosa no verão e seca no inverno. O solo é caracterizado (Santos et al. 2006) como Argissolo Vermelho eutrófico típico, de textura arenosa/média, A moderado, que apresentava, respectivamente, nos horizontes A e B, os seguintes atributos: a) teor de argila de $90 \mathrm{~g} \mathrm{~kg}^{-1}$ e $194 \mathrm{~g} \mathrm{~kg}^{-1}$; b) $\mathrm{pH}$ em $\mathrm{CaCl}_{2}$ de 6,3 e 5,9; c) teor de $\mathrm{P}$ de $75 \mathrm{mg} \mathrm{dm}^{-3}$ e $12 \mathrm{mg} \mathrm{dm}^{-3}$; d) matéria orgânica de $14,0 \mathrm{~g} \mathrm{dm}^{-3} \mathrm{e}$ $8,4 \mathrm{~g} \mathrm{dm}^{-3}$; e) K $\mathrm{K}^{+}$de $2,1 \mathrm{mmol}_{\mathrm{c}} \mathrm{dm}^{-3}$ e 2,0 $\mathrm{mmol}_{\mathrm{c}} \mathrm{dm}^{-3}$; f) $\mathrm{Ca}^{+2}$ de $32 \mathrm{mmol} \mathrm{dm}^{-3}$ e $\left.13 \mathrm{mmol}_{\mathrm{c}} \mathrm{dm}^{-3} ; \mathrm{g}\right) \mathrm{Mg}^{+2}$ de $16 \mathrm{mmol}_{\mathrm{c}} \mathrm{dm}^{-3}$ e $\left.9 \mathrm{mmol}_{\mathrm{c}} \mathrm{dm}^{-3} ; \mathrm{h}\right) \mathrm{H}^{+}+\mathrm{Al}^{+3}$ de $12 \mathrm{mmol}_{\mathrm{c}} \mathrm{dm}^{-3}$ e $13 \mathrm{mmol}_{\mathrm{c}} \mathrm{dm}^{-3}$; i) $\mathrm{Al}^{+3} \mathrm{de} 0 \mathrm{mmol}_{\mathrm{c}} \mathrm{dm}^{-3}$ e $0 \mathrm{mmol}_{\mathrm{c}} \mathrm{dm}^{-3}$; j) soma de bases de $46,4 \mathrm{mmol}_{\mathrm{c}} \mathrm{dm}^{-3}$ e $24,4 \mathrm{mmol}_{\mathrm{c}} \mathrm{dm}^{-3}$; $\mathrm{k}$ ) CTC de $61,1 \mathrm{mmol}_{\mathrm{c}} \mathrm{dm}^{-3} \mathrm{e}$ $37,2 \mathrm{mmol}_{\mathrm{c}} \mathrm{dm}^{-3}$; 1) V\% de $80 \%$ e $63 \%$.

A variedade de laranja estudada foi a Valência (Citrus sinensis (L.) Osbeck), plantada em novembro de 2006, no espaçamento de $7 \mathrm{~m} \mathrm{x} 4 \mathrm{~m}$ entre as plantas (357 plantas ha-1), em um pomar de 3,63 ha. Assim, a malha geoestatística foi alocada em agosto de 2011, constituída por 6 transeções de 228,0 m de comprimento, espaçadas em 14,5 m, em uma área de 1,65 ha. Como cada transeção conteve 20 pontos amostrais, espaçados em 11,4 m, o total deles foi de 120 .

$\mathrm{O}$ atributo pesquisado para a planta foi a produtividade de frutos (PR). Para tanto, foram contadas todas as laranjas (frutos) contidas em cada árvore referente a cada ponto da malha geoestatística e, posteriormente, essa quantidade foi convertida em toneladas ha ${ }^{-1}$. Já os atributos pesquisados para o solo foram derivados da análise química de rotina, 
para fins de fertilidade do solo $\left(\mathrm{P}, \mathrm{MO}, \mathrm{pH}, \mathrm{K}^{+}, \mathrm{Ca}^{2+}\right.$, $\mathrm{Mg}^{2+}, \mathrm{H}^{+}+\mathrm{Al}^{3+}, \mathrm{S}, \mathrm{T}$ e V\%), realizados no laboratório de Fertilidade do Solo da Faculdade de Engenharia de Ilha Solteira (Unesp).

Em 24/08/2011, foram coletados todos os dados representantes do atributo da planta situada mais proximamente do ponto amostral da malha geoestatística. Para tanto, a copa da laranjeira foi dividida, no sentido paralelo ao horizontal, em terços, coletando-se aleatoriamente cinco frutos do terço médio. Efetuou-se a contagem do número total de frutos (NF) e, em seguida, a pesagem da massa média do fruto (em g). Portanto, a produtividade de frutos (PR) foi determinada pelo produto entre o peso médio dos cinco frutos e o NF, transformando-se os dados para $\mathrm{t} \mathrm{ha}^{-1}$.

Em 26/10/2011, foram coletadas amostras deformadas de solo, pelo emprego de um trado de caneca com diâmetro interno de $0,08 \mathrm{~m}$, altura de $0,20 \mathrm{~m}$ e volume de $1,005 \cdot 10^{-3} \mathrm{~m}^{3}$. O local da coleta foi posicionado no centro da entrelinha das laranjeiras, o mais proximamente possível do ponto amostral, à profundidade de $0-0,20 \mathrm{~m}$. As análises químicas de rotina, para fins de fertilidade do solo, foram realizadas conforme Raij et al. (2001), sendo o fósforo em $\mathrm{mg} \mathrm{dm}{ }^{-3}$; matéria orgânica (MO) em $\mathrm{g} \mathrm{dm}^{-3} ; \mathrm{pH}, \mathrm{K}^{+}$, $\mathrm{Ca}^{+2}, \mathrm{Mg}^{+2}, \mathrm{H}^{+}+\mathrm{Al}^{+3}$, soma de bases (S) e capacidade de troca catiônica $(\mathrm{T}) \mathrm{em} \mathrm{mmol}_{\mathrm{c}} \mathrm{dm}^{-3}$ e a saturação por bases $(\mathrm{V} \%)$ em \%.

Foi efetuada a análise descritiva dos atributos, calculando-se a média, mediana, valores mínimos e máximos, desvio-padrão, coeficiente de variação, curtose, assimetria e análise da distribuição de frequência pelo teste de Shapiro e Wilk. Foi montada a matriz de correlação entre todos os atributos pesquisados, contendo todas as combinações pareadas possíveis. Objetivou-se detectar a existência de correlações significativas entre a produtividade de frutos (variável dependente) e os atributos do solo (variáveis independentes). Seguidamente, efetuaram-se regressões lineares simples da produtividade de frutos (PR), em função dos atributos do solo, no intuito de rastrear o qual pudesse funcionar como indicador de qualidade, quando o objetivo fosse aumentar a produtividade da laranja Valência, para o caso em questão.

A análise estatística foi efetuada por meio do programa SAS (Schlotzhaver \& Littell 1997) e de planilha Excel (Lima et al. 2010), enquanto a análise geoestatística foi feita com o programa Gamma Design $\left(\mathrm{GS}^{+}\right.$2004). Para cada atributo, foi analisada a dependência espacial, pelo cálculo do semivariograma isotrópico. Efetuaram-se krigagens e cokrigagens entre a produtividade de frutos e os atributos químicos do solo. Os mapas resultantes, para efeito prático de discussão de suas espacialidades, foram divididos em nove nonos, a saber: central $(\mathrm{C})$, noroeste $(\mathrm{NW})$, norte $(\mathrm{N})$, nordeste $(\mathrm{NE})$, oeste (W), leste (E), sudoeste (SW), sul (S) e sudeste (SE). O objetivo foi ratificar a existência de um atributo (solo) que pudesse espacialmente funcionar como indicador de qualidade, isto é, caracterizar as zonas específicas de manejo, quando o objetivo fosse o de aumentar a referida produtividade.

\section{RESULTADOS E DISCUSSÃO}

A distribuição de frequência normal, representante típica dos dados da planta, é uma condição ideal para análises estatísticas, sejam elas convencional e/ ou geoestatística. Não sendo assim, busca-se a normalidade pela transformação logarítmica (Molin et al. 2007). A PR apresentou distribuição normal, assim como, na maioria dos atributos químicos, ela foi indeterminada ( $\mathrm{P}, \mathrm{MO}, \mathrm{pH}, \mathrm{Ca}^{2+}, \mathrm{Mg}^{2+}, \mathrm{H}^{+}+\mathrm{Al}^{3+}$ e T), com curtose entre 3,302 (T) e -0,395 (MO) e assimetria entre 1,634 (T) e -0,226 (H+Al) (Tabela 1). Ocorreu baixa variabilidade para os dados de $\mathrm{pH}$; média para $\mathrm{MO}, \mathrm{H}+\mathrm{Al}$ e V\%; alta para PR e T; e muito alta para $\mathrm{P}, \mathrm{K}^{+}, \mathrm{Ca}^{2+}, \mathrm{Mg}^{2+}$ e valor $\mathrm{S}$, ficando de acordo com a variabilidade apontada por Oliveira et al. (2009), para o pH, MO e K de um Argissolo cultivado com laranjeira. Especificamente em relação à produtividade média do fruto (PR) do presente estudo $\left(52,0 \mathrm{~kg} \mathrm{pl}^{-1}\right)$, tal valor ficou abaixo dos $59,8 \mathrm{~kg} \mathrm{pl}^{-1}$ obtidos por Molin \& Mascarin (2007), assim como acima dos $46,0 \mathrm{~kg} \mathrm{pl}^{-1}$ obtidos por Oliveira et al. (2009).

Ainda que o solo caracterizado tenha apresentado elevado teor de nutrientes (elevada CTC e $\mathrm{V} \%$ ), a produtividade média de laranjas foi baixa $\left(52,0 \mathrm{~kg} \mathrm{pl}^{-1}\right)$, muito provavelmente devido às severas condições de compactação nas quais o solo se encontrava, uma vez que o controle das plantas daninhas foi efetuado com sucessivas gradagens pesadas, assim como devido ao baixo teor de matéria orgânica do horizonte A arenoso.

Para as correlações significativas, destacaram-se os seguintes pares de atributos: 1) PR x P (r = $0,212 *)$; 2) PR x pH $\left(\mathrm{r}=0,288^{* *}\right)$; 3) PR x Ca $(\mathrm{r}=$ $\left.0,199^{*}\right)$; 4) PR x Mg $\left(\mathrm{r}=0,234^{*}\right)$. Tais correlações, com coeficientes de correlação baixos e positivos, 
Tabela 1. Análise descritiva inicial da produtividade da laranja Valência e de atributos químicos de um Argissolo Vermelho eutrófico (Nova Canaã Paulista, SP, 2011).

\begin{tabular}{|c|c|c|c|c|c|c|c|c|c|c|}
\hline \multirow{3}{*}{ Atributo } & \multicolumn{10}{|c|}{ Medidas estatísticas descritivas } \\
\hline & \multirow{2}{*}{ Média } & \multirow{2}{*}{ Mediana } & \multicolumn{2}{|c|}{ Valor } & \multirow{2}{*}{$\begin{array}{c}\text { Desvio- } \\
\text { Padrão }\end{array}$} & \multicolumn{3}{|c|}{ Coeficiente } & \multicolumn{2}{|c|}{$\begin{array}{c}\text { Probabilidade } \\
\text { do teste }\end{array}$} \\
\hline & & & Mínimo & Máximo & & $\begin{array}{c}\text { Variação } \\
(\%)\end{array}$ & Curtose & Assimetria & $\operatorname{Pr}<w$ & $\mathrm{DF}$ \\
\hline $\mathrm{PR}\left(\mathrm{t} \mathrm{ha}^{-1}\right)$ & 18,577 & 18,471 & 8,793 & 31,524 & 4,267 & 23,0 & 0,454 & 0,387 & 0,181 & $\mathrm{NO}$ \\
\hline $\mathrm{P}\left(\mathrm{mg} \mathrm{dm}^{-3}\right)$ & 8,400 & 7,000 & 1,000 & 30,000 & 5,600 & 67,2 & 1,674 & 1,055 & $10^{-4}$ & $\mathrm{IN}$ \\
\hline $\operatorname{MO}\left(\mathrm{g} \mathrm{dm}^{-3}\right)$ & 12,500 & 13,000 & 10,000 & 16,000 & 1,300 & 10,4 & $-0,395$ & 0,148 & $10^{-4}$ & $\mathrm{IN}$ \\
\hline $\mathrm{pH}$ & 5,900 & 5,800 & 5,300 & 6,900 & 0,400 & 6,4 & $-0,300$ & 0,407 & 0,003 & $\mathrm{IN}$ \\
\hline $\mathrm{K}\left(\mathrm{mmol}_{\mathrm{c}} \mathrm{dm}^{-3}\right)$ & 2,010 & 1,900 & 0,700 & 3,800 & 0,680 & 34,1 & $-0,241$ & 0,357 & 0,079 & NO \\
\hline $\mathrm{Ca}\left(\mathrm{mmol}_{\mathrm{c}} \mathrm{dm}^{-3}\right)$ & 19,600 & 18,000 & 7,000 & 43,000 & 6,800 & 34,4 & 2,744 & 1,518 & $10^{-4}$ & $\mathrm{IN}$ \\
\hline $\mathrm{Mg}\left(\mathrm{mmol}_{\mathrm{c}} \mathrm{dm}^{-3}\right)$ & 9,600 & 9,000 & 5,000 & 20,000 & 2,900 & 30,2 & 1,174 & 1,104 & $10^{-4}$ & $\mathrm{IN}$ \\
\hline $\mathrm{H}+\mathrm{Al}\left(\mathrm{mmol}_{\mathrm{c}} \mathrm{dm}^{-3}\right)$ & 11,800 & 12,000 & 7,000 & 16,000 & 1,600 & 13,7 & 0,554 & $-0,226$ & $2.10^{-4}$ & $\mathrm{IN}$ \\
\hline $\mathrm{S}\left(\mathrm{mmol} \mathrm{dm}^{-3}\right)$ & 31,460 & 29,050 & 14,800 & 64,600 & 9,870 & 31,4 & 0,177 & 0,501 & 0,041 & $\mathrm{TL}$ \\
\hline $\mathrm{T}\left(\mathrm{mmol}_{\mathrm{c}}^{\mathrm{c}} \mathrm{dm}^{-3}\right)$ & 43,770 & 42,000 & 29,000 & 83,800 & 10,200 & 23,3 & 3,302 & 1,634 & $10^{-4}$ & $\mathrm{IN}$ \\
\hline $\mathrm{V}(\%)$ & 71,900 & 70,500 & 58,500 & 92,600 & 7,400 & 10,3 & $-0,306$ & 0,455 & 0,033 & $\mathrm{TL}$ \\
\hline
\end{tabular}

$\mathrm{PR}=$ produtividade de fruto; $\mathrm{DF}=$ distribuição de frequência dos dados, sendo NO, IN e TL, respectivamente, do tipo normal, indeterminada e tendendo a lognormal.

indicaram relação direta entre a PR e os referidos atributos do solo. Sua baixa magnitude (r entre $0,20 *$ e $0,27 * *$ ) deveu-se, basicamente, a dois fatos, previamente comentados por Montanari et al. (2010): a) exemplo clássico de variável dependente (PR) contra independente $(\mathrm{P}, \mathrm{pH}, \mathrm{Ca}$ e $\mathrm{Mg}) ; \mathrm{b})$ elevado número de observações $(\mathrm{n}=120)$. Tal evento é uma realidade, em relação a estudos que normalmente trabalham com muitos dados para a composição de regressões, assim como para a modelagem da dependência espacial (Molin et al. 2007, Andreotti et al. 2010, Montanari et al. 2010, Dalchiavon et al. 2011a e 2011b). Desse modo, as equações ajustadas foram:

$$
\begin{array}{ll}
\mathrm{PR}=17,20+0,161 \mathrm{P} & \left(\mathrm{r}=0,212^{*} ; \mathrm{n}=120\right) \\
\mathrm{PR}=-1,28+3,353 \mathrm{pH} \quad\left(\mathrm{r}=0,288^{*} ; \mathrm{n}=120\right) \\
\mathrm{PR}=16,10+0,126 \mathrm{Ca} \quad\left(\mathrm{r}=0,199^{*} ; \mathrm{n}=120\right) \\
\mathrm{PR}=15,30+0,346 \mathrm{Mg} \quad\left(\mathrm{r}=0,234^{*} ; \mathrm{n}=120\right)
\end{array}
$$

As equações 1 a 4 demonstraram influência linear direta de $\mathrm{P}, \mathrm{pH}, \mathrm{Ca}$ e $\mathrm{Mg}$ sobre a PR. As variáveis independentes ora mencionadas, por não possuírem qualquer relação de interdependência com a variável dependente, ainda que tenham revelado correlações baixas, poderão funcionar como indicadores de qualidade, quando o objetivo for aumentar a produtividade de fruto (PR). Entretanto, em função de ter apresentado o maior coeficiente de correlação $\left(\mathrm{r}=0,288^{* *}\right)$, o modelo ajustado a partir do $\mathrm{pH}$ foi o que mais eficientemente relacionou-se com o aumento da citada produtividade. Portanto, em relação aos valores extremos de $\mathrm{pH}$ presentes na Tabela $1(5,3$ e 6,9), aumentando-se 0,2 unidades de $\mathrm{pH}$ entre tais extremos, poderá haver incremento linear da PR de 0,67 t ha-1, concordando com Dalchiavon et al. (2011a), quando trabalharam com a produtividade do feijoeiro invernal, em função dos teores de matéria orgânica e do valor de $\mathrm{pH}$ do solo, evidenciando, então, haver necessidade de se corrigir o pH do solo em estudo, para proporcionar aumento na produtividade de fruto.

A análise geoestatística (Tabela 2, Figura 1) revelou, para os atributos que não resultaram em efeito pepita puro, coeficientes de correlação espacial $\left(\mathrm{r}^{2}\right)$ muito altos $(0,820-0,933)$, dependências espaciais (ADE) entre médias e muito altas $(50,0-87,2 \%)$ e coeficientes angulares (b) da validação cruzada próximos da unidade. Esses dados foram muito semelhantes àqueles obtidos por Lima et al. (2010) e Montanari et al. (2010), quando foram pesquisadas espécies de eucalipto e feijoeiro, respectivamente, bem como atributos do solo, uma vez que tais trabalhos apresentaram $\mathrm{r}^{2}$ variando de alto a muito alto $(0,670-0,987 \mathrm{e}$ $0,766-0,976)$ e ADE entre média e muito alta $(50,2-$ $90,8 \%$ e $61,6-93,0)$, respectivamente.

Os alcances semivariográficos ficaram entre 24,0 (\#P) e 88,9 m (Ca), concordando com Oliveira et al. (2009), indicando que, para manejos específicos e localizados, os valores referenciais utilizados nos pacotes computacionais empregados na agricultura de precisão não deverão ser inferiores a $24,0 \mathrm{~m}$ (Tabela 2), por representar a distância dentro da qual os valores de um determinado atributo apresentam 
Tabela 2. Parâmetros dos semivariogramas simples e cruzados da produtividade de laranja Valência e de atributos químicos de um Argissolo Vermelho eutrófico (Nova Canaã Paulista, SP, 2011).

\begin{tabular}{|c|c|c|c|c|c|c|c|c|c|c|c|}
\hline \multirow{3}{*}{ Atributo } & \multicolumn{11}{|c|}{ Parâmetros do ajuste } \\
\hline & \multirow{2}{*}{ Modelo } & \multirow{2}{*}{$\mathrm{C}_{\mathrm{o}}$} & \multirow{2}{*}{$\mathrm{C}_{\mathrm{o}}+\mathrm{C}$} & \multirow{2}{*}{$\mathrm{A}_{\mathrm{o}}(\mathrm{m})$} & \multirow{2}{*}{$r^{2}$} & \multirow{2}{*}{ SQR } & \multicolumn{2}{|c|}{$\mathrm{ADE}$} & \multicolumn{3}{|c|}{ Validação cruzada } \\
\hline & & & & & & & $\%$ & Classe & $\mathrm{a}$ & $\mathrm{b}$ & $\mathrm{r}$ \\
\hline \multicolumn{12}{|c|}{ Semivariogramas simples } \\
\hline PR & esf. (175) & 6,48 & 13,00 & 71,1 & 0,863 & 4,17 & 50,0 & ME & 3,56 & 0,806 & 0,387 \\
\hline \#P & gau. (198) & 5,88 & 17,34 & 24,0 & 0,820 & 4,63 & 66,1 & $\mathrm{AL}$ & $-0,08$ & 0,916 & 0,480 \\
\hline MO & esf. (106) & 0,56 & 1,51 & 37,4 & 0,884 & $3,75 \cdot 10^{-2}$ & 62,7 & AL & 1,60 & 0,872 & 0,439 \\
\hline $\mathrm{pH}$ & esf. (214) & $4,62 \cdot 10^{-2}$ & 0,15 & 63,8 & 0,865 & $9,00 \cdot 10^{-4}$ & 69,5 & $\mathrm{AL}$ & 0,07 & 0,989 & 0,669 \\
\hline $\mathrm{K}$ & exp. (214) & $6,30 \cdot 10^{-2}$ & 0,49 & 67,8 & 0,933 & $4,32 \cdot 10^{-3}$ & 87,2 & MA & $-0,04$ & 1,018 & 0,686 \\
\hline $\mathrm{Ca}$ & esf. (214) & 19,40 & 49,10 & 88,9 & 0,884 & 87,70 & 60,4 & $\mathrm{AL}$ & 0,89 & 0,957 & 0,524 \\
\hline $\mathrm{Mg}$ & esf. (110) & 1,22 & 7,79 & 70,3 & 0,851 & 6,53 & 84,3 & MA & 0,05 & 0,996 & 0,728 \\
\hline $\mathrm{H}+\mathrm{Al}$ & gau. (109) & 90,00 & 2,30 & 29,0 & 0,878 & 0,12 & 60,8 & AL & 3,97 & 0,663 & 0,369 \\
\hline S & esf. (200) & 25,50 & 89,00 & 85,0 & 0,913 & $2,83 \cdot 10^{2}$ & 71,4 & $\mathrm{AL}$ & 0,15 & 0,999 & 0,628 \\
\hline $\mathrm{T}$ & esf. (214) & 44,50 & $1,09.10^{2}$ & 83,6 & 0,931 & $2,15.10^{2}$ & 59,3 & ME & 0,62 & 0,988 & 0,548 \\
\hline V\% & esf. (200) & 19,20 & 55,00 & 80,4 & 0,914 & 78,40 & 65,1 & $\mathrm{AL}$ & 4,11 & 0,943 & 0,570 \\
\hline \multicolumn{12}{|c|}{ Semivariogramas cruzados - Planta $x$ Solo } \\
\hline $\mathrm{PR}=\mathrm{f}(\mathrm{MO})$ & gau. (161) & 0,32 & 0,72 & 65,0 & 0,319 & 0,32 & 58,4 & ME & 9,12 & 0,505 & 0,339 \\
\hline $\mathrm{PR}=\mathrm{f}(\mathrm{pH})$ & gau. (175) & $1,00.10^{-3}$ & $4,46.10^{-1}$ & 66,7 & 0,669 & $9,83 \cdot 10^{-2}$ & 99,8 & MA & 8,92 & 0,516 & 0,348 \\
\hline $\mathrm{PR}=\mathrm{f}(\mathrm{Mg})$ & gau. (170) & $1,00.10^{-2}$ & 3,53 & 63,4 & 0,619 & 8,81 & 99,7 & MA & 8,37 & 0,545 & 0,369 \\
\hline
\end{tabular}

PR

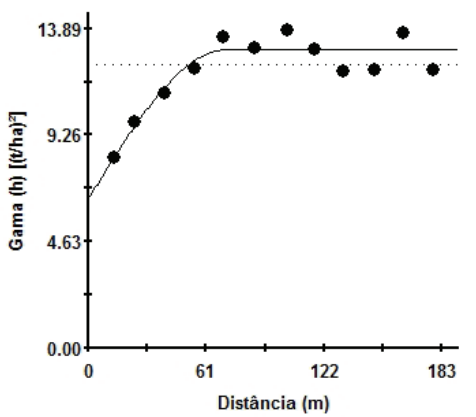

pH

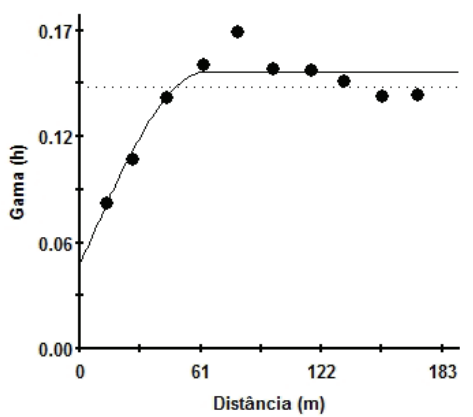

(a)

(b)

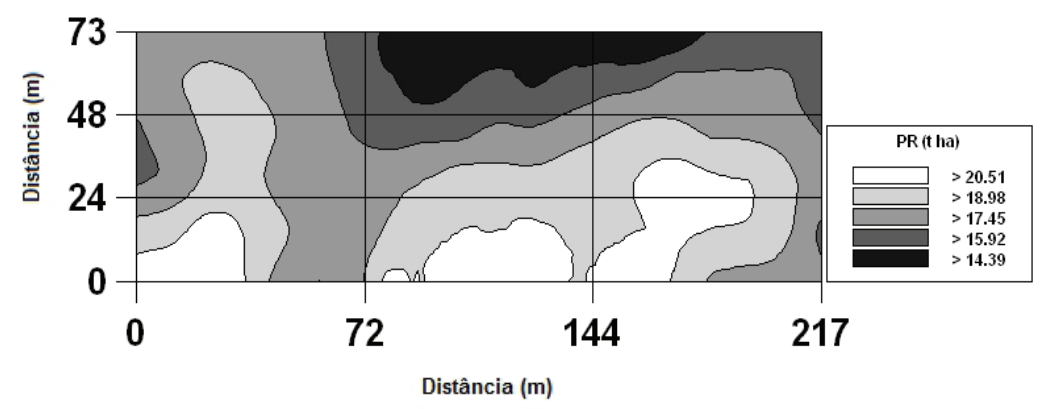

(c)

(d)

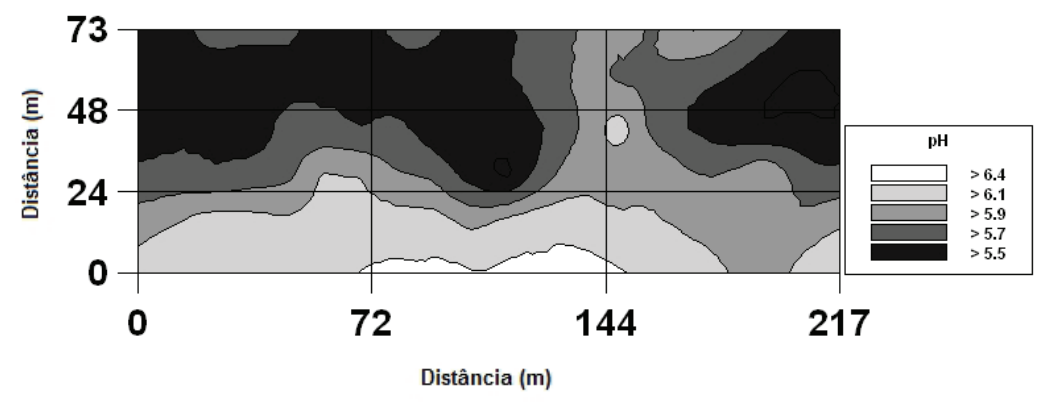

Figura 1. Semivariogramas simples (a, c) e mapas de krigagem (b, d) da produtividade de laranja Valência e do pH de um Argissolo Vermelho eutrófico (Nova Canaã Paulista, SP, 2011). 
correlação espacial entre si, haja vista que o valor do alcance pode influenciar na qualidade da estimativa, uma vez que determina o número de valores usados na interpolação. Assim, estimativas feitas com interpolação por krigagem, utilizando os maiores valores do alcance, tendem a ser mais confiáveis, apresentando mapas que representam melhor a realidade (Dalchiavon et al. 2011b).

Por outro lado, os semivariogramas cruzados (Tabela 2, Figura 2) apresentaram baixo $(0,319)$ e altos $(0,619$ e 0,669$)$ coeficientes de determinação espacial $\left(\mathrm{r}^{2}\right)$, para as variáveis secundárias $\mathrm{MO}, \mathrm{pH}$ e $\mathrm{Mg}$, respectivamente, ficando de acordo com Andreotti et al. (2010), Montanari et al. (2010) e Basso et al. (2011). Assim, do ponto de vista geoestatístico (Figura 1), ocorreram apreciáveis correlações espaciais diretas da PR com o pH do solo, de modo que a cokrigagem $\mathrm{PR}=\mathrm{f}(\mathrm{pH})$ proporcionou, nos sítios de menores valores de $\mathrm{pH}$, as menores $\mathrm{PR}$, sendo o inverso perfeitamente verdadeiro (Figuras 1 e 2).
Portanto, o pH, por ter revelado estreita relação espacial com a PR, teve apreciável desempenho no delineamento de cinco zonas específicas de manejo do solo, para a cultura da laranja Valência, de modo que pode ser utilizado como indicador, quando o objetivo for aumentar a produtividade de frutos de laranja. Esse resultado assemelhou-se ao que foi observado por Dalchiavon et al. (2011a), os quais relataram correlação espacial da produtividade de feijoeiro invernal com o $\mathrm{pH}$ do solo.

Com o auxílio dos dados da Tabela 1, nas Figuras $1 \mathrm{~b}$ e $1 \mathrm{~d}$, puderam ser generalizadas duas zonas específicas de manejo do $\mathrm{pH}$ do solo intimamente associadas à produtividade de laranja, que foram confirmadas pela cokrigagem $\mathrm{PR}=\mathrm{f}(\mathrm{pH})$ da Figura 2, a saber: 1) zona 1, ocupando os seis nonos superiores (NW, N, NE, W, C e E), com pH de 5,3-5,9 e PR de $8,79-17,45$ t ha $^{-1}$; 2) zona 2, ocupando os três nonos inferiores (SW, S, e SE), com pH de 5,9-6,9 e PR de $17,45-31,52 \mathrm{t} \mathrm{ha}^{-1}$. Portanto, como suas produtivida-
$P R=f(p H)(t / h a)$

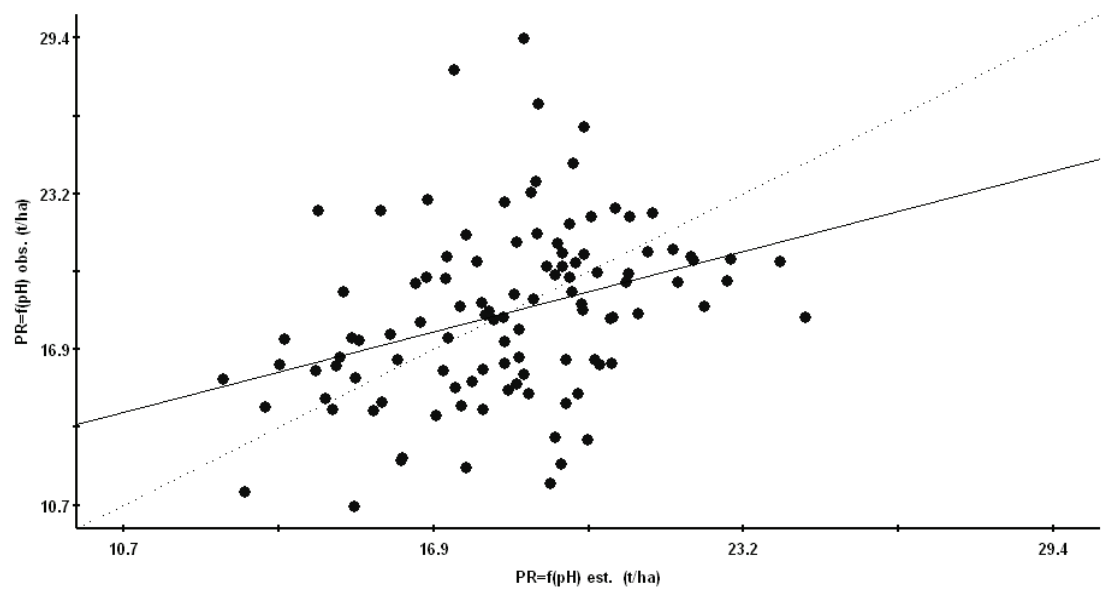

$P R=f(p H)$

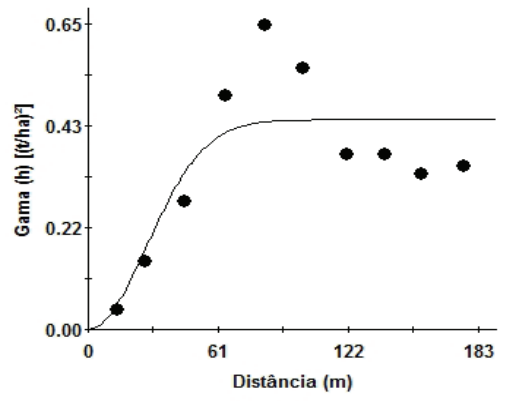

(b)

(c)

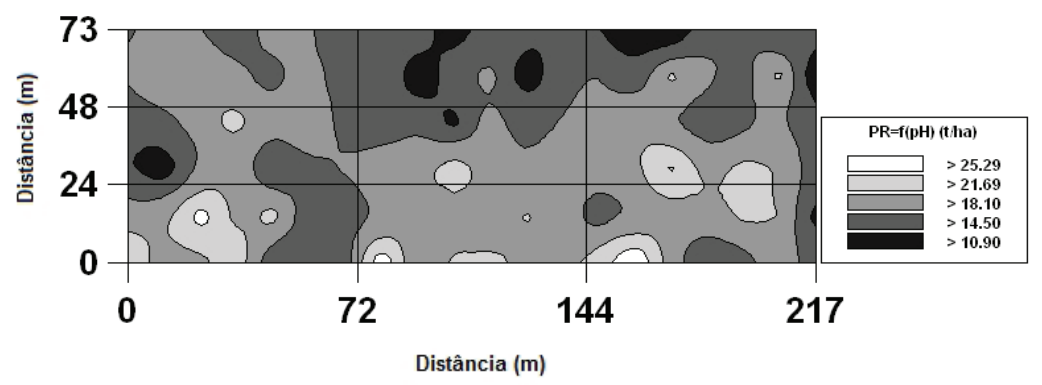

Figura 2. Validação cruzada (a), semivariograma cruzado (b) e mapa de cokrigagem (c) da produtividade de laranja Valência (PR), em função do pH de um Argissolo Vermelho eutrófico (Nova Canaã Paulista, SP, 2011). 
des médias foram respectivamente de $13,12 \mathrm{tha}^{-1} \mathrm{e}$ $24,49 \mathrm{t} \mathrm{ha}^{-1}$, a produtividade global da área seria de $16,91 \mathrm{tha}^{-1}\left[\left(6 / 9 \times 13,12 \mathrm{tha}^{-1}\right)+\left(3 / 9 \times 24,49 \mathrm{tha}^{-1}\right)\right]$. Contudo, para se obter a produtividade média de $24,49 \mathrm{t} \mathrm{ha}^{-1}$, típica da zona 2 , seria necessário proporcionar um valor médio do $\mathrm{pH}$ do solo, à zona 1 , entre 5,9-6,9, para que sua produtividade fosse igualada à da zona 2. Dessa maneira, verifica-se, no mapa, a presença de manchas com produtividades variadas (5,3-31,52 $\left.\mathrm{t} \mathrm{ha}^{-1}\right)$, corroborando o que já havia sido constatado por Farias et al. (2003), Molin \& Mascarin (2007) e Oliveira et al. (2009), os quais trabalharam com o mapeamento da produtividade em pomares cítricos e concluíram que os mapas de produtividade apresentaram alta variabilidade espacial.

Portanto, visando ao aumento da PR, devem ser envidados esforços para que, de maneira geral, na área pesquisada, o $\mathrm{pH}$ tenha valores entre 5,9 e 6,9, para que a PR assuma seus mais elevados valores, que ficarão entre 21,69 t ha ${ }^{-1}$ e 25,29 tha ${ }^{-1}$ (Figura 2). Assim, face ao exposto, é evidente a importância da adoção de técnicas de manejo localizado, que vise à geração de mapas de produtividade em área de citros, para a implantação de um sistema de agricultura de precisão que facilite o gerenciamento integrado de entradas e saídas no sistema agrícola, concomitantemente à avaliação dos efeitos de adoção de taxas variadas de insumos.

Dessa forma, a krigagem atestou a criação de zonas específicas de manejo do solo: uma com adequado $\mathrm{pH}$ e maior produtividade e outra com o inverso. Futuramente, essa metodologia, que preconiza a aplicação de insumos com taxas variáveis, poderá ser aplicada a pomares a serem instalados com o intuito de se aumentar a lucratividade agrícola.

\section{CONCLUSÕES}

1. A produtividade de laranja resultou em significativas cokrigagens ordinárias, tanto com o $\mathrm{pH}$ como com o teor de $\mathrm{Mg}$ do solo utilizado na pesquisa. Assim, a aplicação dessa técnica possibilitou a identificação de duas zonas específicas de manejo do $\mathrm{pH}$ do solo intimamente associadas à referida produtividade.

2. A identificação de duas zonas específicas de manejo ratificou a importância da agricultura de precisão, frente ao aumento da produtividade de laranja. Isso porque, mantendo-se as duas zonas específicas de manejo observadas, a produtividade global da área seria de $16,91 \mathrm{t} \mathrm{ha}^{-1}$. Contudo, proporcionando-se condições ótimas de $\mathrm{pH}$ do solo àquela de maior acidez, poder-se-ia obter um incremento de $7,58 \mathrm{t} \mathrm{ha}^{-1}$ na produtividade de frutos, alcançando a média global de $24,49 \mathrm{t} \mathrm{ha}^{-1}$.

\section{REFERÊNCIAS}

ANDREOTTI, M. et al. Produtividade da soja correlacionada com a porosidade e a densidade de um Latossolo Vermelho do Cerrado brasileiro. Ciência Rural, Santa Maria, v. 40, n. 3, p. 520-526, 2010.

BASSO, F. C. et. al. Relações entre produtividade de sorgo forrageiro e atributos físicos e teor de matéria orgânica de um latossolo do Cerrado. Pesquisa Agropecuária Tropical, Goiânia, v. 41, n. 1, p. 135-144, 2011.

BRASIL. Ministério da Agricultura, Pecuária e Abastecimento. Citrus. 2012. Disponível em: $<$ http:// www.agricultura.gov.br/vegetal/culturas/citrus $>$. Acesso em: 07 abr. 2012.

DALCHIAVON, F. C. et al. Variabilidade espacial da produtividade do feijoeiro correlacionada com atributos químicos de um Latossolo Vermelho distroférrico sob sistema de semeadura direta. Bragantia, Campinas, v. 70, n. 4, p. 1-9, 2011a.

DALCHIAVON, F. C. et al. Produtividade da soja e resistência mecânica à penetração do solo sob sistema plantio direto no Cerrado brasileiro. Pesquisa Agropecuária Tropical, Goiânia, v. 41, n. 1, p. 8-19, 2011 b.

FARIAS, P. R. S. et al. Agricultura de precisão: mapeamento da produtividade em pomares cítricos usando geoestatística. Revista Brasileira de Fruticultura, Jaboticabal, v. 25, n. 2, p. 235-241, 2003.

GAMMA DESIGN SOFTWARE $\left(\mathrm{GS}^{+}\right)$. Geostatistics for the environmental sciences. 7. ed. Plainwell: Gamma Design, 2004.

LIMA, C. G. R. et al. Atributos físico-químicos de um latossolo do Cerrado brasileiro e sua relação com características dendrométricas do eucalipto. Revista Brasileira de Ciência do Solo, Viçosa, v. 34, n. 1, p. 163173, 2010.

MOLIN, J. P.; MASCARIN, L. S. Colheita de citros e obtenção de dados para mapeamento da produtividade. Engenharia Agrícola, Jaboticabal, v. 27, n. 1, p. 259-266, 2007.

MOLIN, J. P. et al. Variação espacial na produtividade de milho safrinha devido aos macronutrientes e à população 
de plantas. Revista Brasileira de Milho e Sorgo, Sete Lagoas, v. 6, n. 3, p. 309-324, 2007.

MONTANARI, R. et al. Aspectos da produtividade do feijão correlacionados com atributos físicos do solo sob elevado nível tecnológico de manejo. Revista Brasileira de Ciência do Solo, Viçosa, v. 34, n. 6, p. 1811-1822, 2010.

OLIVEIRA, P. C. G. et al. Variabilidade espacial de propriedades químicas do solo e da produtividade de citros na Amazônia Oriental. Revista Brasileira de Engenharia Agrícola e Ambiental, Campina Grande, v. 13, n. 6, p. 708$715,2009$.
RAIJ, B. V. et al. Análise química para avaliação da fertilidade de solos tropicais. Campinas: Instituto Agronômico, 2001.

SANTOS, H. G. et al. Sistema brasileiro de classificação de solos. 2. ed. Rio de Janeiro: Embrapa Solos, 2006.

SCHLOTZHAVER, S. D.; LITTELL, R. C. SAS system for elementary statistical analysis. 2. ed. Cary: SAS Institute, 1997.

TSCHIEDEL, M.; FERREIRA, M. F. Introdução à agricultura de precisão: conceitos e vantagens. Ciência Rural, Santa Maria, v. 32, n. 1, p. 159-163, 2002. 